DANUBIA $36^{\text {th }}$ Danubia-Adria Symposium on Advances in Experimental Mechanics
D
A

\title{
STRAIN ANALYSIS OF CORD-RUBBER COMPOSITES USING DIC
}

\author{
Thomas LEHMANN $^{1}$, Jörn IHLEMANN ${ }^{1}$ \\ 1 Chemnitz University of Technology, Professorship of Solid Mechanics, Reichenhainer Straße 70, \\ 09126 Chemnitz, Germany, E-mail: thomas.lehmann@mb.tu-chemnitz.de
}

\section{Introduction}

Cord-rubber composites are applied in various technical applications, e.g. tires, sleeves, air springs and belts. The development of such components include numerical simulations, which require the knowledge of the material's characteristic described by suitable material models with respective material parameters [1]. Therefore, experimental tests for material characterization, parameter identification as well as validation of numerical models are necessary. Stressing of cord reinforced elastomers can result in strong inhomogeneous stress and strain fields. The experimental detection of the resulting large strain gradients requires high-performance measuring methods. In this contribution, 3D Digital Image Correlation (DIC) is used to determine the 3D coordinates, followed by the evaluation of strain fields on the surface of cord reinforced rubber specimens by means of a postprocessing procedure.

\section{Experimental setup, procedure}

Experiments were carried out using specimens with rubber matrix material (special composition of Vibracoustic AG \& Co. KG) variable reinforced by polyamide cords, aramid cords or hybrid cords (consisting of polyamide and aramid yarns) with the cord angles $\varphi$ of $10^{\circ}$ and $35^{\circ}$. The geometry of the specimen was designed as a sleeve with the nominal dimensions: inner diameter $95 \mathrm{~mm}$, sleeve thickness $1.5 \mathrm{~mm}$, initial free length $75 \mathrm{~mm}$. The specimen is clamped at the top and bottom sides, see Fig. 1. In order to visualize maximum strain differences on the surface of the specimens, a configuration without elastomer top layer was used. The experiments were performed in a servohydraulic test rig, which can be used for tension, compression and torsion tests, at Helmut-Schmidt-Universität / Universität der Bundeswehr Hamburg, see Fig. 1. The first step of the test procedure was the application of an initial inner pressure of 1 bar. Subsequent, axial compression in steps of $10 \mathrm{~mm}$ until $s=50 \mathrm{~mm}$ (nominal values) with following torsion by $\alpha= \pm 1^{\circ}$ in every compression step was performed. The inner pressure was kept constant during the whole tests. An example of a loaded specimen is given in Fig. 2.

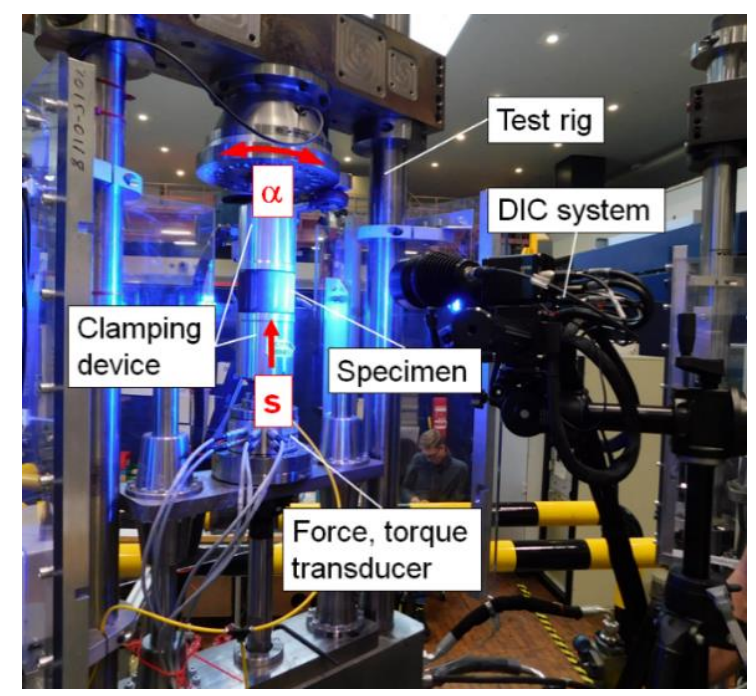

Fig. 1. Experimental setup of the tests.

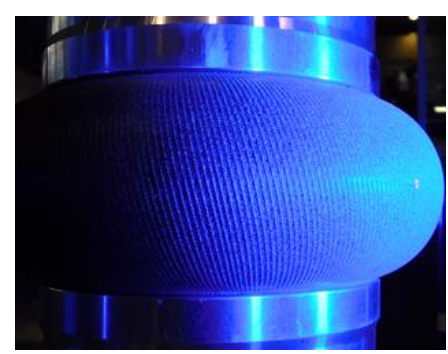

Fig. 2. Loaded specimen (cord angle $10^{\circ}$ ).

In the presented investigations, the 3D DIC system Gom Aramis 4M was applied using a small measuring volume with horizontal and vertical dimensions of $25 \times 18 \mathrm{~mm}$ (nominal values). The specimens were coated with the required fine speckle patterns by means of white paint (achieving enough contrast to the dark surface of the specimens). Considering the limited depth of field using the small measuring volume, an out-of-planetracking of the cameras was performed since significant displacements of the analyzed section in this direction occured. This adaption was carried out in the respective loading steps to get well-focused images for the DIC. For strain evaluation, the 3D coordinates determined by DIC were used. 


\section{Evaluation method}

Strains regarding tangential directions of the surface based on the 3D coordinates were calculated by means of a developed evaluation procedure using Matlab. Hereinafter, $\tilde{\mathbf{u}}$ and $\tilde{\mathbf{v}}$ (see Fig. 3) represent the local directions of curvilinear coordinate lines on the curved surface in the reference state. The DIC data was approximated by B-spline surfaces [2],[3]. The two-dimensional deformation gradient $\mathbf{F}$ regarding the tangential directions, which is calculated considering the curved geometry in the reference and the deformed state, was determined using the approximated spline functions of the 3D coordinates. As a strain measure, the Langrangian Hencky strain tensor was determined, which is given by:

$$
\mathbf{H}=0.5 \ln \left(\mathbf{F}^{\mathrm{T}} \mathbf{F}\right) .
$$

Furthermore, the Green-Langrange strain tensor (based on the deformation gradient $\mathbf{F}$ and the identity tensor $\mathbf{I}$ ) is calculated by:

$$
\gamma=0.5\left(\mathbf{F}^{\mathrm{T}} \mathbf{F}-\mathbf{I}\right) \text {. }
$$

Calculating $\gamma$ for transformed (rotated) evaluation directions $(\tilde{\xi}, \tilde{\boldsymbol{\eta}}$, see Fig. 3) following the orientation of cord reinforcement (by angle $\varphi$, reference state), the shear angle $\theta_{\xi \eta}$ regarding these transformed directions is determined using:

$$
\theta_{\xi \eta}=\arcsin \left(\frac{2 \gamma_{\xi \eta}}{\sqrt{1+2 \gamma_{\xi \xi}} \sqrt{1+2 \gamma_{\eta \eta}}}\right) .
$$

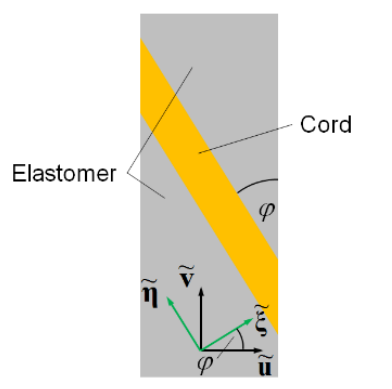

Fig. 3. Evaluation directions.

\section{Results}

In Fig. 4, an example of the evaluation for the maximum load step (without torsion) for polyamide reinforced rubber, cord angle $35^{\circ}$ is given. At the left side the Hencky strain $H_{u u}$ is shown. Large strain differences between cord and elastomer sections are well detected, where the strain in the cords is approximately zero. In addition, the distribution of the shear angle $\theta_{\xi \eta}$ is demonstrated (Fig. 4, right). The evaluation shows that the shear deformation is concentrated in the elastomer sections.

Furthermore, the actual direction of the elastomer and the cord sections can be reconstructed by means of the distribution of the deformations.

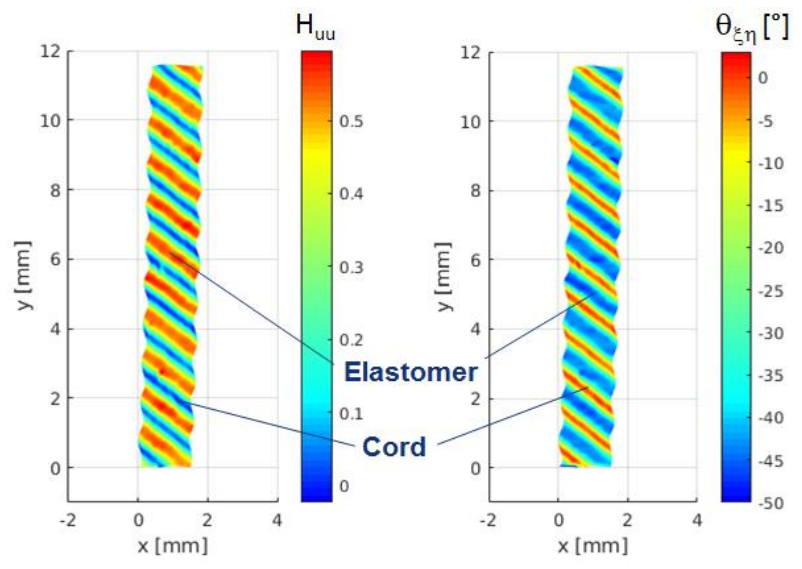

Fig. 4. $H_{u u}$ (left) and $\theta_{\xi \eta}$ (right) (strip at the specimen's middle), axial compression of $s=47.7 \mathrm{~mm}$.

\section{Conclusions}

Special sleeve specimens of cord-rubber composites were loaded by inner pressure, compression and torsion. In order to achieve a data base for validation of numerical simulations, experimental deformation analyses of the specimen surfaces were carried out. By means of 3D DIC and a special evaluation procedure, the challenging detection of the resulting large strain differences was successfully performed. As expected, the deformations are concentrated in the elastomer sections.

\section{Acknowledgements}

The authors gratefully acknowledge the financial support from ContiTech AG, Mehler Engineered Products $\mathrm{GmbH}$, Goodyear S.A. and Vibracoustic AG \& Co. KG.

\section{References}

[1] Donner, H. FEM-basierte Modellierung stark anisotroper Hybridcord-Elastomer-Verbunde, $\mathrm{PhD}$ Thesis, 2017.

[2] Hoschek, J., Lasser, D. Grundlagen der geometrischen Datenverarbeitung, second ed., Teubner, Stuttgart, 1992.

[3] Lehmann, T., Müller, J., Ihlemann, J. DIC deformation analyses of $\mathrm{Mg}$ specimens at elevated temperatures, Mater. Today-Proc., 2018, 5, 2677826783. 